\title{
The Effects of a Physical Education Intervention to Support the Satisfaction of Basic Psychological Needs on the Motivation and Intentions to Be Physically Active
}

\author{
by \\ Evelia Franco ${ }^{1}$, Javier Coterón ${ }^{1}$
}

\begin{abstract}
The aim of the study was to investigate the effects of an intervention to support the basic psychological needs on the satisfaction of these needs, intrinsic motivation, intention to be physically active and some enjoyment-related outcomes in Physical Education. The present study incorporated strategies presented by Standage and Ryan (2012) in a previous study. A quasi-experimental study was conducted with two groups (nexperimental $=30$; $n_{\text {control }}=23$ ) of $2 n d$ year Secondary Education students aged between 13 and $15(M=13.35, S D=.62)$ by delivering 24 physical education classes. The teacher in the experimental group underwent prior and continual training. The results revealed that the students from the experimental group showed a significant increase in the perception of autonomy and competence. Furthermore, the experimental group showed a greater perception than the control group in the enjoyment related to learning and contents. These results provide information about the efficacy of an intervention programme based on the strategies presented by Standage and Ryan (2012) to foster satisfaction of basic psychological needs and facilitate support for basic psychological needs to promote the development of positive learning-related outcomes.
\end{abstract}

Key words: intervention effects, learning, adolescents, motivation.

\section{Introduction}

Several studies have shown the physical and psychological benefits of the practice of physical activity for children and young people as well as dangers that a lack of exercise can create for physical, psychological and social health (Gray and Leyland, 2008). However, the amount of time physical activity is practiced rarely reaches the general recommendations for these groups, and an important drop in physical activity levels during adolescence has been observed in recent years (Verloigne et al., 2012). Under these circumstances, encouraging active and healthy lifestyles has become an educational and health priority in different countries (World Health Organization, 2011) leading to a growing interest in the study of strategies that promote the aforementioned lifestyles.
Physical education (PE) is a mandatory activity for everyone during the ages in which children exhibit high levels of susceptibility to abandoning physical activity. Therefore, the PE class is an attractive field for the analysis of physical activity-related variables, such as physical activity intentions (Hein et al., 2004; Sun and Chen, 2010b). These intentions can be a good indicator of children's motivation towards physical activity as well as a strong predictor of future behaviours (Shephard and Trudeau, 2000). Findings from these and other studies reveal an association between motivational patterns within the PE contexts and exercise-related behaviours (Lochbaum and Jean-Noel, 2016).

Self-determination theory (SDT; Deci and Ryan, 1985) provides insight as to how motivational processes affect students'

1 - Universidad Politécnica de Madrid, Spain. 
engagement in school PE. SDT consists of six mini-theories. One of these is the basic psychological needs theory, according to which humans have the following three basic psychological needs (BPN): the innate needs for autonomy, competence and relatedness (Deci and Ryan, 2000). The need for autonomy is fulfilled when people perceive that they are the origin of their choices and decisions and that they are acting in accord with their integrated sense of self. Competence concerns an individual's need to feel a sense of mastery through effective interaction with their environment. The third need, relatedness, corresponds to feeling securely attached to and being respected by significant others. Satisfaction of these psychological needs is assumed to directly enhance the psychological and physical well-being (Deci and Ryan, 2000). Another mini-theory is the organismic integration theory, which considers that behaviour is voluntary or self-determined and distinguishes a continuum of motivation from the most selfdetermined (intrinsic motivation) to the least selfdetermined behaviour (amotivation). SDT states that intrinsic motivation is driven by the desire to satisfy the aforementioned BPN (Deci and Ryan, 2000). According to the Hierarchical Model of Motivation (Vallerand, 2001), social contextual variables can influence the BPN satisfaction, which, in turn, might predict the person's motivation quality. Furthermore, this model suggests that greater levels of self-determination could enhance positive consequences.

In the context of $\mathrm{PE}$, the type of motivation that has more frequently been associated with adaptive patterns is intrinsic motivation. This type of motivation seems to be related to desirable outcomes, such as enjoyment (Pulido et al., 2014), positive attitudes towards physical activity (Halvari et al., 2011), physical activity practice (Halvari et al., 2011) and intention to be physically active (Pulido et al., 2014; Taylor et al., 2010).

Based on previous studies that showed a positive relationship between granting autonomy and intrinsically motivating students during PE classes (Taylor and Ntoumanis, 2007), interventions have been focused on fostering support to students' autonomy during classes (González-Cutre et al., 2014; Shen et al., 2009). However, based on what SDT postulates, satisfying the three BPNs, not only the need for autonomy, is fundamental, as each BPN plays an important and complementary role for development and optimal experience, as well as for daily well-being (Deci and Ryan, 2000). Therefore, studies on the support for the three needs in a PE context have been conducted over the last few years (Amado et al., 2014; Cheon et al., 2012).

Aside from motivational processes, attitudes and perceptions towards PE may influence adolescents' participation in $\mathrm{PE}$ at school as well as organized sports outside school (Kjønniksen et al., 2009). According to the literature, enjoyment can be seen as one of the most important affective consequences of quality $\mathrm{PE}$ and has been proposed to be an important factor increasing physical activity in PE and leisure time within children and adolescents (Cairney et al., 2012; Wang et al., 2010).

Considering the above, it would seem reasonable to develop strategies which may lead to enhance the aforementioned motivational variables as well as enjoyment and learning outcomes in in the PE context.

Different recommendations have been provided to transfer the tenets of BPN into practice (Deci and Vansteenkiste, 2004). One of the most current and complete approaches aiming to gather the existing scientific evidence to create a needs supportive environment was proposed by Standage and Ryan (2012). Although they considered the situational components that provided support for each basic need and offered some practical suggestions to be applied in exercise and physical activity settings in previous studies, few studies have considered this approach in the selection of strategies to be implemented to foster BPN (Gillison et al., 2014). To foster support for autonomy, they provide strategies such as maximizing the opportunity for choice, minimizing ego involvement, and vocalizing in an appropriate manner. Additionally, to foster support for competence, they recommend facilitating activities that provide an optimal challenge, minimizing task involvement, and providing ample positive feedback. Finally, strategies such as the provision of cooperation and relational support (through "exercise buddy" schemes) and the involvement of others are provided to foster support for 
relatedness. Taking into consideration the nature of the PE-context, our study consisted of the design and development of an intervention based on the strategies suggested by Standage and Ryan (2012) that could be most properly implemented in this type of setting (Figure 1).

The present study sought to analyse the effect that an intervention based on the aforementioned strategies for fostering autonomy, competence and relatedness satisfaction had on the BPN satisfaction, intrinsic motivation, and the intention to be physically active. Furthermore differences in the perceptions of enjoyment between control and experimental groups were analysed: learning, contents, teacher's performance, activities and liking compared to other previous curricula contents practiced during the term.

These practical applications were based on the hypothesis that environments that were supportive of psychological needs would enhance autonomous motivation and optimal psychological functioning (Deci and Ryan, 2008). Therefore, this study hypothesized that students in the experimental group would display increased BPN satisfaction, intrinsic motivation, and intention to be physically active. Additionally, it was expected that the intervention group would show greater perceptions of learning, enjoyment of the contents, teacher's performance and activities than the control group.

\section{Methods}

\section{Participants}

The sample comprised 53 students (30 males and 23 females) from two Secondary Education school classes in Madrid (Spain) between the ages of 13 and 15 years $(M=13.35$, $\mathrm{SD}=.62$ ). The classes participating in the study were randomly selected among those from that educational level in which the teacher taught. All of the students were in the $2^{\text {nd }}$ year of secondary education. One class with 23 students was assigned to a control group, and another class with 30 students was assigned to an experimental group. Students who were absent from PE classes for more than three days (12.5\% of the program) were excluded from the study. The percentage of eliminated sample was lower than $5 \%$, with a participation rate of $96.4 \%$ and with 2 invalidated questionnaires (3.6\%) out of a total of 55 collected.

\section{Measures}

Psychological needs.

The Basic Psychological Needs in Exercise Scale (BPNES; Vlachopoulos and Michailidou, 2006), adapted for a Spanish context (Sánchez and Núñez, 2007), was used to measure the satisfaction of basic psychological needs. The instrument was preceded by the heading "During my Physical Education classes..." followed by 12 items (four per factor), which measured the satisfaction of autonomy (e.g., "the way the exercises are carried out coincides perfectly with the way in which I want to do them"), satisfaction of competence (e.g., " I feel that I have progressed greatly with respect to the final objective that I had set out for myself"), and the satisfaction of relatedness (e.g., "I feel very comfortable when I carry out the exercises with the other mates"). Responses to the questionnaire were closed and rated on a 5-point Likert scale ranging from 1 (strongly disagree) to 5 (strongly agree). The internal consistency of the instrument, measured by Cronbach's alpha, was adequate for both the pre-test and the post-test (pre/post autonomy: $.87 / .76$; pre/post competence: $.71 / .75$; pre/post relatedness: .89/.91).

Intrinsic motivation.

The Intrinsic Motivation factor of the Spanish version (Moreno et al., 2009) of the Perceived Locus of Causality (PLOC; Goudas et al., 1994) was used. The instrument was preceded by the heading "I take part in my Physical Education class...", and four of the items measured intrinsic motivation (e.g., "because Physical Education is fun"). Responses to this questionnaire were closed and rated on a 5-point Likert scale ranging from 1 (strongly disagree) to 5 (strongly agree). The internal consistency of the factor was adequate (Cronbach's alpha of .87 for both measures).

Intention to be physically active.

The adapted and translated Spanish version (Moreno et al., 2007) of the Intention to be Physically Active Questionnaire (Hein, et al., 2004) was used. It is composed of five items for measuring the subject's intention for being physically active (for example, "I am interested in developing my physical fitness"). The items are preceded by the phrase "Regarding your intention to partake in sports...". The questions were responded to using a Likert scale that ranged 
from 1 (strongly disagree) to 5 (strongly agree). The scale showed a Cronbach's alpha of .79 in the pretest and .76 in the postest.

\section{Enjoyment.}

An ad hoc questionnaire was designed to measure the enjoyment experienced by the students. It was composed of 5 items which asked about liking for learning achieved ("I feel I learnt how to play rugby and I enjoyed it"), the content ("I like rugby"), the teacher's performance ("I liked the way the teacher developed the lessons"), activities ("I liked the activities we have practiced in these PE lessons") as well as the enjoyment they had during the activities in relation to other contents taught before the intervention ("I enjoyed the rugby's lessons... than the rest of activities we have done in PE for this year"). The first three questions were responded to using a Likert scale that ranged from 1 (strongly disagree) to 5 (strongly agree). In the last item, students could choose among "much more", "more", "as much", "less", "much less" to complete the sentence. The scale showed a Cronbach's alpha of .87. This questionnaire had been previously validated in a sample of 246 PE students $(\mathrm{M}=14.35$; $\mathrm{SD}=1.41)$. CFA results showed an adequate adjustment for the one factor's structure $\left(\chi^{2}=15.74 ; p=.01 ; \chi^{2} / \mathrm{g} .1\right.$. $=3.15 ; \mathrm{CFI}=.97 ; \mathrm{TLI}=.94 ; \mathrm{RMSEA}=0.9 ; \mathrm{SRMR}=$ $0.4)$. Factor loads ranged between .51 and .86 .

\section{Design and Procedures}

A pre-post, quasi-experimental design was used in which the participants were divided into the control and experimental groups. As there were two natural classes already established by the school centre, it was not possible to incorporate randomization. The independent variable for the study was the support of autonomy, competence and relatedness in the experimental condition. The study previously received the approval of the Ethics Committee of the Technical University of Madrid. All participants were treated in agreement with the ethical guidelines of the American Psychological Association (2002) with respect to consent, confidentiality and anonymity of their answers. Moreover, informed written consent was obtained from the parents and the head teachers of the school centres on behalf of the minor participants involved in the study.

The intervention was implemented over
24 PE sessions lasting for three months. Questionnaires were administered during the PE class by a member of the research group. This person explained the importance that the results would have on their future physical education classes and emphasized that the anonymity of the participants would be maintained as well as encouraged them to provide their most honest answers to the questions. Students completed the questionnaire in the classroom without the presence of the teacher and in a climate that enabled them to concentrate without any type of distraction; it lasted for 25 minutes. Data were collected during the sessions right before and after the intervention in both groups.

The contents had been designed according to the PE programme of the School Department. Intervention was carried out in the unit of Rugby. Both groups would engage in the same objectives as well as the same activities. Every session was described in detail in the previous programme.

Therefore the contents and activities of the School Department were maintained. However, changes in methodology were applied in the experimental condition according to the aforementioned strategies (Standage and Ryan, 2012). To maintain compliance with the established PE programme, a list of specific actions to facilitate each of the strategies was first established. These actions were grouped as follows: (a) the role of the teacher, (b) the role of the students, and (c) the activity structure and development. Second, a dossier was prepared on the sessions to be implemented in which modifications were assigned to each of the activities depending on their characteristics.

Control group

The control group followed the contents programmed by the School Department. The sessions in this group were taught by the school's PE teacher, who had seven years of work experience, in accordance with his methodology. This teacher had conducted PE classes for both groups the previous year, assuring similar teaching experiences for all of the students. The study's objective was explained to the teacher who did not receive any type of training or specific information that could affect the development of the sessions in the control group. There was no contact between the control and 
experimental groups during the intervention.

Experimental group teacher training

Before the intervention, the teacher for this group attended a training programme built upon two parts.

Strategies to support basic psychological needs. This part lasted 10 hours and was grouped into five $2 \mathrm{~h}$ sessions. Contents were distributed in the following sections: (a) general postulates of SDT emphasizing those related to basic needs satisfaction, (b) motivational strategies to promote learning environments that would support autonomy, competence and relatedness satisfaction; and (c) contributions of experimental works that had been previously developed.

Practical training. On the basis of the dossier previously created, the teacher designed and developed four sessions in an external class group. These sessions were videotaped and analysed by the teacher and two PE experts to strengthen the implemented strategies, detect possible problems and clarify doubts.

All along the intervention four randomized selected sessions were videotaped. In order to ensure that strategies were implemented as it had been planned to do in advance, a control list of the strategies was created and the two PE expert researchers fulfilled it separately. The intra-class correlation coefficients were high (ICC $>0.92$ ).

\section{Statistical Analysis}

A Kolmogorov-Smirnov test was performed to verify the normality of the data and showed that it was non-parametric $(p<.05)$. Thus, a Mann-Whitney test was performed to analyse possible differences between the groups before the intervention. Then, the main analysis was performed to investigate the intervention effects in two ways. First, to verify the intra-group differences between the pre-test and post-test data collection, a Wilcoxon test was performed with each of the groups. Next, a new Mann-Whitney test was conducted to analyse the inter-group differences between the two groups after the intervention. The effect sizes of the comparisons were estimated using Cliff's delta. The SPSS 20.0 software program was used to process the data.

\section{Results}

\section{Preliminary analysis}

The results showed significant differences in the satisfaction of autonomy $(U=183.50, p<.01$, Cliff's delta $=-.42)$, the satisfaction of competence $(U=243.00, p<.05$, Cliff's delta $=-.28)$, the satisfaction of relatedness $(U=206.50, p<.01$, Cliff's delta $=-.37)$, and intrinsic motivation $(U=$ 208.50, $p<.01$, Cliff's delta $=-.37$ ). The control group reflected higher scores than the experimental group in all of these variables (Table 1). Effects of the intervention

Table 1 shows the scores of each dependent variable both before and after the intervention as well as the $\mathrm{Z}$ scores and Cliff's deltas. In the experimental group, significant differences were found both in satisfaction of autonomy and competence $(p<.05)$. The scores on both variables were higher in the post-test. Conversely, in the control group, a significant difference was found in the satisfaction of competence $(p<.05)$. Students in this group showed a higher satisfaction of competence before the intervention.

Strategies to Support BPN Satisfaction

\begin{tabular}{ll} 
& Strategies to Support BPN Satisfaction \\
\hline \multirow{3}{*}{ Autonomy } & A1: Maximizing opportunity for choice \\
& A2: Acknowledging students' feelings \\
& A3: Minimizing ego involvement \\
\hline \multirow{3}{*}{ Competence } & C1: Provision for optimal challenge \\
& $\begin{array}{l}\text { C2: Ample administration of positive feedback } \\
\text { C3: Fostering of task involvement in activities }\end{array}$ \\
\hline \multirow{2}{*}{ Relatedness } & R1: Acknowledging students' feelings \\
& R2: Supporting an "exercise buddy" scheme \\
\hline
\end{tabular}

Figure 1

Strategies selected to support BPN satisfaction (from Standage and Ryan, 2012). 


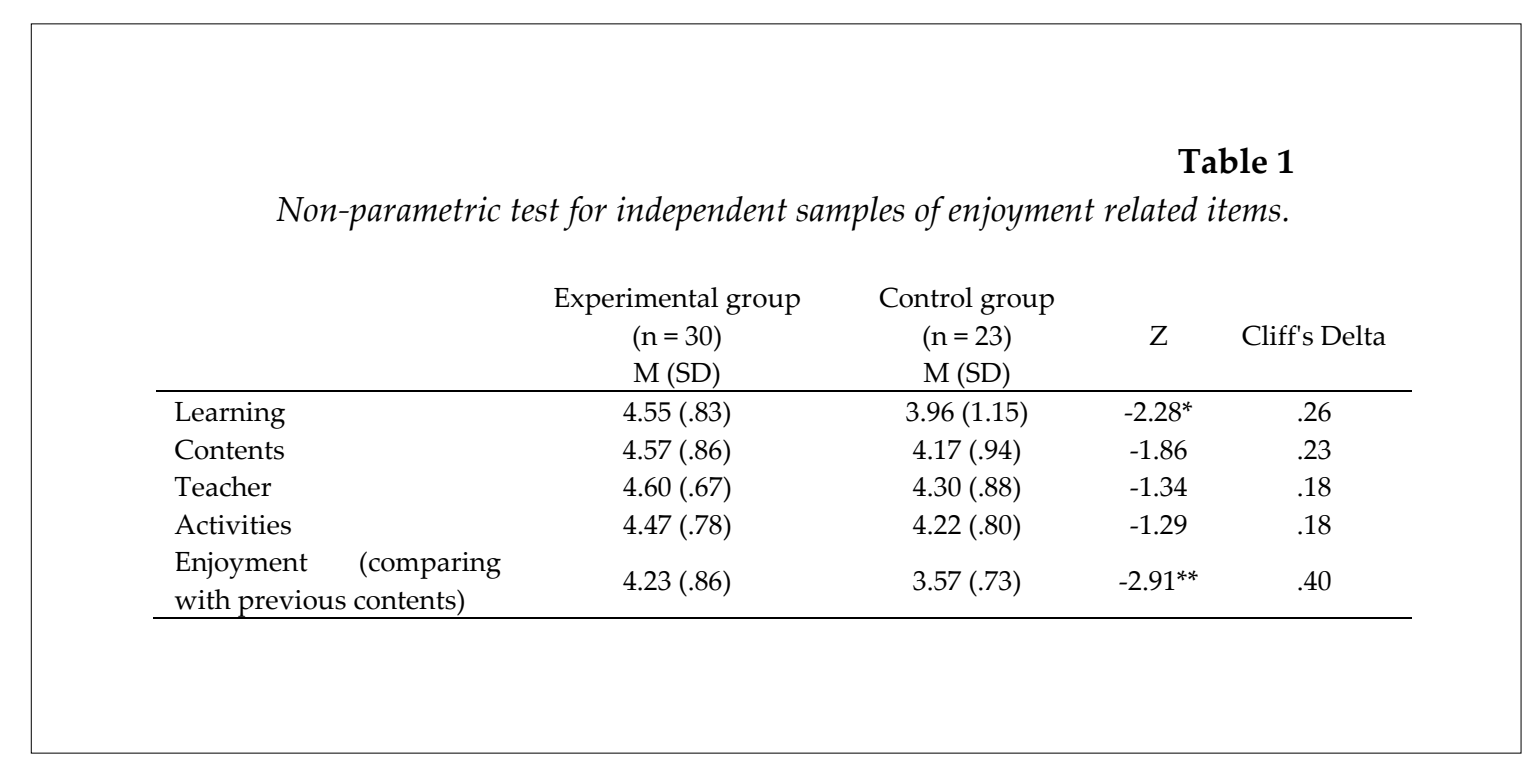

\begin{tabular}{|c|c|c|c|c|c|c|c|}
\hline \multicolumn{8}{|c|}{$\begin{array}{c}\text { Table } 2 \\
\text { Non-parametric test for related samples of basic psychological needs, } \\
\text { intrinsic motivation and practice intention. }\end{array}$} \\
\hline & & \multicolumn{3}{|c|}{ Experimental group $(\mathrm{n}=30)$} & \multicolumn{3}{|c|}{ Control group $(n=23)$} \\
\hline & & $\mathrm{M}(\mathrm{SD})$ & Z & $\begin{array}{l}\text { Cliff's } \\
\text { Delta }\end{array}$ & $\mathrm{M}(\mathrm{SD})$ & $\mathrm{Z}$ & $\begin{array}{l}\text { Cliff's } \\
\text { Delta }\end{array}$ \\
\hline \multirow[t]{2}{*}{ Autonomy } & Pre & & $-2.26^{*}$ & .31 & & -.59 & .08 \\
\hline & Post & $3.53(.73)$ & & & $3.92(.57)$ & & \\
\hline \multirow[t]{2}{*}{ Competence } & Pre & $3.76(.85)$ & $-2.77^{*}$ & .38 & $4.18(.57)$ & $-2.60^{*}$ & .35 \\
\hline & Post & $4.13(.60)$ & & & $3.93(.59)$ & & \\
\hline \multirow[t]{2}{*}{ Relatedness } & Pre & $3.81(1.04)$ & -.64 & .09 & $4.35(.89)$ & -.97 & .13 \\
\hline & Post & $3.81(1.01)$ & & & $4.28(.59)$ & & \\
\hline \multirow[t]{2}{*}{ Intrinsic Motivation } & Pre & $3.88(1.05)$ & -.70 & .10 & $4.55(.58)$ & -1.37 & .19 \\
\hline & Post & $4.17(.95)$ & & & $4.41(.66)$ & & \\
\hline \multirow[t]{2}{*}{ Practice Intention } & Pre & $4.19(.90)$ & -1.61 & .22 & $4.48(.49)$ & -1.11 & .15 \\
\hline & Post & $3.97(.83)$ & & & $4.33(.72)$ & & \\
\hline
\end{tabular}

Significant differences were found between the groups after the intervention. The control group showed higher scores in the satisfaction of autonomy ( $\mathrm{U}=230.50, \mathrm{p}<.05$, Cliff's delta $=-.28)$.
Regarding the other variables related to the learning process, the experimental group showed significantly lower levels of previous knowledge about the contents that were developed and 
higher levels of learning and enjoyment during the intervention (Table 2).

\section{Discussion}

The objective of this study was to test the effects of an intervention implemented in a PE class that fostered the support for the satisfaction of the BPNs, intrinsic motivation and the intention to be physically active. We conducted a programme based on the guidelines of Standage and Ryan (2012). This study hypothesized that students in the experimental group would display increased BPN satisfaction, intrinsic motivation, and intention to be physically active. Additionally, it was expected that the intervention group would show greater perceptions of enjoyment.

In this study, the groups were nonhomogenous with prior differences in some of the dependent variables. This fact must be assumed since in educational settings the groups are classes that have been previously established by the school and this cannot be modified (Amado et al., 2014; González-Cutre et al., 2014). The control group showed greater BPN satisfaction and intrinsic motivation. After the intervention, we only found significant differences in the autonomy satisfaction. The elimination of the differences after the intervention was due to an increase in the scores of intrinsic motivation in the experimental group. With regard to competence, differences disappeared due to an increase in the experimental group and a decrease in the control group. Lastly, differences in relatedness were eliminated exclusively because of a decrease in the control group.

Understanding the results through the lens of the SDT, the changes observed in the experimental group in the variable of autonomy satisfaction suggest that the present PE intervention could increase student perceptions of autonomy support. As SDT postulates that autonomy support is one of the most important social factors in explaining motivational processes (Deci and Ryan, 1985), this finding was expected. Several studies agree that there are positive effects of interventions fostering autonomy support or BPN support on autonomy satisfaction among students (Amado et al., 2014; Cheon et al., 2012; González-Cutre et al., 2014). Although to a lesser extent, there are also studies that have found evidence of the effect of these types of interventions on competence satisfaction (Cheon et al., 2012; Shen et al., 2009).

Regarding competence, the groups showed unequal trends in the present study. On the one hand, the scores decreased in the control group. The fact that students faced contents that they had not experienced previously in PE could have made it more difficult for the students in the control group to perceive themselves as competent. As different theoretical frameworks in relation to competence postulate, time is necessary to notice a substantial change when starting to learn new tasks (Bandura, 1990), indicating that subjects must be given sufficient time so that they can have enough successful experiences to make them feel competent. Research conducted with similar characteristics has noted the lack of significant changes in the perception of competence when implementing short programmes fostering BPN (Amado et al., 2014). However, in the present study, competence satisfaction increased after the intervention in the experimental group, which could be due to the changes implemented. Evidence of the positive effect of this type of strategy on the perception of competence can be found in the existing literature (Amado et al., 2014; Cheon et al., 2012). However, it would be interesting for future studies to examine how the previous knowledge of the contents affects the students' perceived competence.

With respect to the need for relatedness, although it was expected based on previous studies (Amado et al., 2014; Cheon et al., 2012; Shen et al., 2009), no increase occurred in the satisfaction of this need in the experimental group. The lack of significant change in this variable could be affected by two different aspects. First, class groups remained invariable since the previous school year. This might have contributed to creating stable relationships that were difficult to modify through this type of intervention. Second, it is important to note that the strategies used to foster the need for relatedness were mainly based on "the buddy scheme". The presence of activities considering this strategy could not have been enough during the intervention. It would be appropriate to test whether relatedness satisfaction could be enhanced by performing an intervention in which 
"the buddy scheme" was used more often.

Regarding intrinsic motivation, an increase occurred in the satisfaction of this variable in the experimental group with respect to the results found in the control group, but the values were not significant. This positive trend could have been caused by the gain in autonomy and competence perception. The fact that students felt more autonomous and that they had some level of control in the PE classes, as well as more competent to perform the new tasks, could have led to the emergence of intrinsic reasons to engage in PE lessons. Previous studies in the literature have shown a positive effect of this type of intervention on intrinsic motivation through the enhancement of the perception of autonomy (Shen et al., 2009).

The experimental condition in this study did not result in a change in students' intentions to be physically active in the future in spite of previous studies testing the association between motivational processes in the PE class and the promotion of extra-curricular physical activity (González-Cutre et al., 2014), which suggested that levels of intention to be active in the future could be expected to improve in the experimental group. Cheon et al. (2012) assessed the effectiveness of an intervention to help PE teachers to be more supportive of autonomy during instruction. In that study, it was found that, although no differences emerged in the participants' intentions to be physically active in the first phase of the study, subjects in the experimental group reported higher intentions after completing the whole programme. In this study, the effect of the intervention on BPN satisfaction was perceived after the first phase. This suggests that, while there are certain motivational variables susceptible to being modified in a short period of time, it could be that attitude modifications involving behaviour changes need intervention programmes of longer duration.

The experimental group showed a higher perception of learning, suggesting that contexts in which students feel autonomous and responsible for some decisions related to their own educational process may facilitate learning. To a lesser extent, some studies have examined the influence of autonomy support on learning. Shen et al. (2009), in a study conducted with a sample of adolescents, found that perceived autonomy support by teachers significantly predicted students' need satisfaction adjustment and led to learning achievement. The findings of our study support this association. However, results published by Sun and Chen (2010a), from a study developed with similar participants, revealed that intrinsic motivation contributed little to knowledge and skill achievement. In the attempt to demonstrate the connections between selfdetermined motivation and student learning in physical education, the findings of the present study reveal the positive effect of a BPN supportive intervention in the perception of learning. However, as it has been previously suggested (Sun and Chen, 2010b), further research should be conducted to clarify these associations. The results also showed that the students in the experimental group perceived greater enjoyment for the contents taught during the intervention than the control group when comparing these sessions to the previous contents taught in PE. The findings of the study, in line with those of previous studies (Pulido et al., 2014), suggest the decisive role that supportive needs satisfaction interventions have on the emergence of positive affective outcomes, such as enjoyment, which, in turn, could lead to enhanced physical activity levels among adolescents (Wang et al., 2010).

This study presents some limitations that should be mentioned. The first limitation is related to the size of the study groups. The small group sizes were due to the difficulties of conducting an intervention study in a structured educational context. It would be interesting to use a larger sample size in future studies, which would allow us to apply predictive statistical techniques, such as structural equation modelling, to determine whether the changes produced in any variables may be due to changes produced by another variable, as we have hypothesized with regard to intrinsic motivation. In addition to the sample size limitation, the differences found between the groups before the intervention as well as the presence of two different teachers could have had an effect on the final results. It would be useful if future conditions of groups were standardized.

The present study has allowed us to better understand the influence that changes in some didactical aspects of the PE class have on 
students' motivation. The main conclusion drawn is the usefulness of an intervention that incorporates strategies aimed at supporting BPN on autonomy satisfaction, competence satisfaction and intrinsic motivation. Also to be considered is the fact that there was no effect of the intervention on the satisfaction of relatedness, an aspect that could be more difficult to modify as the class groups usually remain stable along the educational stage, and the intention to be physically active, a variable that could require longer interventions to be modified.
Despite some design limitations, such as the size of the sample and the length of the intervention, the findings of the present study intend to be a small contribution to support the associations between self-determined motivation and PE context outcomes through experimental design studies (Sun and Chen, 2010b; Van den Berghe et al., 2014). In this respect, this study adds to the existing literature an example of implementation of strategies fostering basic psychological needs as well as its outcomes in a real PE setting.

\section{Acknowledgements}

The authors would like to thank the financial support to the project entitled "Improving our teenagers' health for a future life: motivation in physical education", provided by the Universidad Politécnica de Madrid (Spain) [grant number AL14-PID-40].

\section{References}

Amado D, Del Villar F, Leo, FM, Sánchez-Oliva D, Sánchez-Miguel PA, García-Calvo T. Effect of a multidimensional intervention programme on the motivation of physical education students. PLOS one, 2014; 9: e85275

American Psychological Association. Ethical principles of psychologists and code of conduct (Amended June 2, 2010). American Psychological Association; 2002

Bandura A. Perceived self-efficacy in the exercise of personal agency. J Appl Sport Psychol, 1990; 2: 128-163

Cairney J, Kwan M, Velduizen S, Hay J, Bray SR, Faught BE. Gender, perceived competence and the enjoyment of physical education in children: a longitudinal examination. Int J Behav Nutr Phys Act, 2012; 9: 26

Cheon SH, Reeve J, Moon IS. Experimentally based, longitudinally designed, teacher-focused intervention to help physical education teachers be more autonomy supportive toward their students. J Sport Exercise Psy, 2012; 34: 365-396

Deci EL, Ryan RM. Intrinsic motivation and self-determination in human behaviour. New York: Plenum; 1985

Deci EL, Ryan RM. The "What" and "Why" of goal pursuits: Human needs and the self-determination of behavior. Psychol Inq, 2000; 11: 227-268

Deci EL, Ryan RM. Facilitating optimal motivation and psychological well-being across life's domains. Can Psychol, 2008; 49: 14-23

Deci EL, Vansteenkiste M. Self-determination theory and basic need satisfaction: Understanding human developmant in positive psychology. Riserche di Psichologi, 2004; 1: 23-40

Gillison F, Standage M, Verplanken B. A cluster randomised controlled trial of an intervention to promote healthy lifestyle habtis to school leavers: study rationale, design, and methods. Public Health, 2014; 14: $221-228$

González-Cutre D, Ferriz R, Beltrán-Carrillo VJ, Andrés-Fabra JA, Montero-Carretero C, Cervelló E, MorenoMurcia JA. Promotion of autonomy for participation in physical activity: a study based on the transcontextual model of motivation. Educ Psychol, 2014; 34: 367-384 
Goudas M, Biddle S, Fox K. Perceived locus of causality, goal orientations, and perceived competence in school physical education classes. Brit J Educ Psychol, 2004; 64: 453-463

Gray L, Leyland AH. Overweight status and psychological well-being in adolescent boys and girls: a multilevel analysis. Eur J Public Health, 2008; 18: 616-621

Halvari H, Skjesol K, Bagoien TE. Motivational climates, achievement goals, and physical education outcomes: A longitudinal test of achievement goal theory. Scan J Educ Res, 2011; 55: 79-104

Hein V, Müür M, Koka A. Intention to be physically active after school graduation and its relationship to three types of intrinsic motivation. Eur Phys Educ Rev, 2004; 10: 5-19

Kjonniksenn L, Fjortoft I, Wold B. Attitude to physical education and participation in organized youth sports during adolescence related to physical activity in young adulthood: A 10-year longitudinal study. Eur Phys Educ Rev, 2009; 15: 139-154

Lochbaum MR, Jean-Noel J. Perceived autonomy-support instruction and student outcomes in physical education and leisure-time: A meta-analytic review of correlates. Ricyde, 2016; 12: 29-47

Moreno JA, González-Cutre D, Chillón M. Preliminary validation in Spanish of a scale designed to measure motivation in physical education classes: The perceived locus of causality (PLOC) scale. Span J Psychol, 2009; 12: 327-337

Moreno JA, Moreno R, Cervelló E. The physical self-concept as a predictor of the intention of being physically active. Psicología y Salud, 2007; 17: 261-267

Pulido JJ, Sánchez-OlivaD, Amado D, González-Ponce I, Sánchez-Miguel PA. Influence of motivational processes on enjoyment, boredom and intention to persist in young sportspersons. S Afr J Res Sport Physic Educ Recreation, 2014; 36: 135-149

Sánchez J, Núñez JL. Psychometric properties of the Spanish version of the scale of basic psychological needs in physical exercise. Preliminary Analysis. Rev Iberoam Psicol Ejercicio Deporte, 2007; 2: 83-92

Shen B, McCaughtry N, Martin J, Fahlman M. Effects of teacher autonomy support and students' autonomous motivation on learning in physical education. Res Q Exercise Sport, 2009; 80: 44-53

Shephard RJ, Trudeau F. The legacy of physical education: influences on adult lifestyle. Pediatr Exerc Sci, 2000; $12: 34-50$

Standage M, Ryan RM. Self-Determination theory and exercise motivation: Facilitating self-regulatory processes to support and maintain health and well-being. In Roberts G, Treasure DC. Advances in motivation in sport and exercise. Champaign, IL: Human Kinetics, 233-270; 2012

Sun H, Chen A. An Examination of Sixth Graders' Self-Determined Motivation and Learning in Physical Education. J Teach Phys Educ, 2010a; 29: 262-277

Sun $\mathrm{H}$, Chen A. A pedagogical understanding of the self-determination theory in physical education. Quest, 2010b; 62: 364-384

Taylor IM, Ntoumanis N. Teacher motivational strategies and student self-determination in physical education. J Educ Psychol, 2007; 99: 747-760

Taylor IM, Ntoumanis N, Standage M, Spray C. Motivational predictors of physical education students' effort, exercise intentions, and leisure-time physical activity: a multilevel linear growth analysis. J Sport Exercise Psy, 2010; 32: 99-120

Vallerand R. A hierarchical model of intrinsic and motivation in sport and exercise. In Roberts G. Advances in motivation in sport and exercise. Champaign, IL: Human Kinetics, 263-320; 2001

Van den Berghe L, Vansteenkiste M, Cardon G, Kirk D, Haerens L. Research on self-determination in physical education: key findings and proposals for future research. Phys Educ Sport Pedagog, 2014; 19: 97-121 
Verloigne M, Lippevelde WV, Maes L, Yildrim M, Chinapaw M, Manios Y, Androutsos O, Kovács E, Bringolf-Isler B, Brug J, De Bourdeaudhuij I. Levels of physical activity and sedentary time among 10- to 12- year-old boys and girls across 5 European countries using accelerometers: an observational study within the ENERGY-project. Int J Behav Nutr Phys Act, 2012; 9: 34

Vlachopoulos S, Michailidou S. Development and initial validation of a measure of autonomy, competence and relatedness: the Basic Psychological Needs in Exercise Scale. Meas Phys Educ Exerc Sci, 2006; 10: $179-201$

Wang CG, Liu WC, Chatzisarantis N. Lim, BSC. Influence of perceived motivational climate on achievement goals in physical education: A structural equation mixture modeling analysis. J Sport Exercise Psy, 2010; 32: 324-338

World Health Organization. Promoting sport and enhancing health in European Union countries. Copenhague, Denmark: World Health Organization; 2011

\section{Corresponding author:}

\section{Javier Coterón López}

Facultad de Ciencias de la Actividad Física y del Deporte-INEF

Calle Martín Fierro, 7 CP 28040 MADRID

Phone: 913364120

E-mail address: j.coteron@upm.es 